\title{
Whole-Body Heating Decreases Skin Vascular Response to Low Orthostatic Stress in the Lower Extremities
}

\author{
Fumio YAMAZAKI, Yoshiro NAKAYAMA, and Ryoko SONE \\ Department of Clinical Pathophysiology, School of Health Sciences, University of Occupational and Environmental Health, \\ Kitakyushu, Japan; Department of Exercise and Health Sciences, Faculty of Education, University of Yamaguchi, Japan
}

\begin{abstract}
To elucidate the influence of heat stress on cutaneous vascular response in the lower extremities during orthostatic stress, a head-up tilt (HUT) test at angles of $15^{\circ}, 30^{\circ}, 45^{\circ}$, and $60^{\circ}$ for 4 min each was conducted under normothermic control conditions followed by whole-body heat stress produced by a hot water-perfused suit in healthy volunteers. Skin blood flows (SkBF) in the forearm, thigh, and calf were monitored using laser-Doppler flowmetry throughout the experiment. Furthermore, to elucidate the effects of increased core and local skin temperatures on the local vascular response in calf skin under increasing orthostatic stress, the thigh was occluded at 20,30,50,70, and $80 \mathrm{mmHg}$ with a cuff in both the normothermic condition and the whole-body or local heating condition. Significant decreases in forearm SkBF during HUT were observed at an angle of $60^{\circ}$
\end{abstract}

during normothermia and at $30^{\circ}$ or more during heating. SkBF in the thigh and calf was decreased significantly by HUT at $15^{\circ}$ and above during normothermia, and there was no significant reduction of SkBF in these sites during HUT at the lower angles $\left(15^{\circ}-\right.$ $45^{\circ}$ ) during whole-body heating. Significant decreases of calf SkBF were observed at cuff pressures of $20 \mathrm{mmHg}$ and above during normothermia and of $30 \mathrm{mmHg}$ and above during wholebody and local heating, respectively. These results suggest that SkBF in the lower extremities shows a marked reduction compared with the upper extremities during low orthostatic stress in normothermia, and the enhanced skin vasoconstrictor response in the lower extremities is diminished by both whole-body and local heat stress.

Key words: skin blood flow, head-up tilt, baroreflex, local reflex, central blood volume.

In humans, exposure to a hot environment increases the skin blood flow (SkBF) and sweat rate, thus increasing heat dissipation, and results in a reduction of central blood volume [1-3]. The change in the distribution of blood with heat stress alters the level of loading in baroreceptors during a given postural challenge, which therefore may modulate the peripheral vascular response to orthostatic stress [4]. A neural control of the cutaneous vascular response can be important in the maintenance of arterial blood pressure during orthostatic stress, when the skin represents a significant fraction of the total vascular conductance during heat stress $[1,5,6]$. Previous reports showed that baroreceptor unloading induced by lower body negative pressure (LBNP) elicits cutaneous vasoconstriction in the forearm via the active vasoconstrictor system in normothermia, but largely via the active vasodilator system in hyperthermia [5, 7]. To date, however, most findings about the cutaneous vascular response to orthostatic stress were obtained from studies of the forearm at the level of the heart, and information from the other areas such as the calf is limited. It is important to know the characteristics of the vascular response in the lower extremities to orthostatic stress, because vascular constriction in the lower extremities during orthostatic stress can contribute to a decrease of blood pooling in the lower body, in addition to an increase of peripheral resistance [3].

Cutaneous vascular response in the lower body during orthostatic stress may differ from that in the forearm at the heart level, because cutaneous vasoconstriction in the extremities in a dependent position during orthostasis can be induced by local reflex (i.e., venoarteriolar response) in addition to baroreflex [3,8-11]. With regard to the local reflex response, it has been reported that local heating to $42^{\circ} \mathrm{C}$ significantly attenuated cutaneous venoarteriolar response in the forearm [12]. Thus it is speculated that the local reflex control of cutaneous vasomotion in the lower body may be attenuated by heat stress, but information on the thermal modulation in the local reflex has been restricted. For example, whether an increased core $\left(T_{\text {core }}\right)$ or local skin temperature $\left(T_{\mathrm{sk}}\right)$ during whole-body heat stress is needed for the modulation is unknown. If an increased $T_{\text {core }}$ is needed for the thermal modulation of local reflex in the lower body, the modulation may be centrally driven.

Received on Jan 11, 2006; accepted on Apr 10, 2006; released online on Apr 12, 2006; doi:10.2170/physiolsci.RP000406 Correspondence should be addressed to: Fumio Yamazaki, Department of Clinical Pathophysiology School of Health Sciences, Univ. of Occupational and Environmental Health (UOEH), 1-1 Iseigaoka, Yahatanishi-ku, Kitakyushu, 807-8555 Japan. Phone: +81-93-6917270, Fax: +81-93-691-7127, E-mail: yamazaki@health.uoeh-u.ac.jp 
But if an increased local temperature is required for thermal modulation, a direct effect of temperature on the local response may be suggested. Since orthostatic tolerance decreases in a hot environment compared with a cool environment [13-16], the clarification of the characteristics of cutaneous vasomotor control in the lower body during orthostatic stress in heat-stressed humans is also important.

In the present study, we first examined cutaneous vascular responses in the forearm at heart level and the lower extremities (thigh and calf) during a graded head-up tilt (HUT) as an orthostatic stress during normothermia and whole-body heating (Protocol 1). Next we examined the effect of increased $T_{\text {core }}$ on the cutaneous vascular response in the calf to increases of calf venous pressure in a supine position (Protocol 2). During this protocol, $T_{\text {sk }}$ at the sites where SkBF was measured in the calf was maintained at a neutral level. We then examined the effect of local warming on cutaneous vascular response in the calf to increases of calf venous pressure (Protocol 3). The results from protocols 2 and 3 may enable us to identify the roles of increased $T_{\text {core }}$ and local $T_{\text {sk }}$ on the local reflex resulting from increased venous pressure in the calf during HUT.

\section{METHODS}

Subjects. A total of 11 male and 11 female volunteers participated in the experiments. Their average age was 21 \pm 0 (SE) yr, average weight $57.7 \pm 2.4 \mathrm{~kg}$, and average height $166 \pm 2 \mathrm{~cm}$. All subjects were healthy nonsmokers with no history of cardiovascular disease. Written informed consent was obtained after a thorough explanation of the present study, including its purpose and risks. The experiments were approved by the Ethics Committee of Medical Care and Research of the University of Occupational and Environmental Health.

Experimental procedures and protocols. Three experiments consisting of different protocols were conducted. In each one, the subjects were tested under conditions of normothermia followed by whole-body or local heating. They were equipped with sensor probes and thermocouples in a climatic chamber $\left(26^{\circ} \mathrm{C}\right.$ ambient temperature, $50 \%$ relative humidity). The protocols are as follows.

Protocol 1: This protocol was performed to examine the cutaneous vascular responses in the arms and legs to a graded HUT. Eight male volunteers participated in the study. The subjects wore a tube-lined water-perfused suit that covered the entire body except for the head and right arm. The mean $T_{\mathrm{sk}}$ was controlled by changing the water temperature of the suit and maintained at $\sim 33^{\circ} \mathrm{C}$ in normothermia, then increased to $\sim 38^{\circ} \mathrm{C}$ during whole-body heating. HUT tests, which consisted of 4 min each of supine rest (baseline control), and $15^{\circ}, 30^{\circ}, 45^{\circ}$, and $60^{\circ}$ HUT were progressively carried out in each thermal condition.
During the tests, both arms and hands rested at heart level on shelves fixed to the tilt table. Care was taken to ensure that the subjects were completely relaxed by instructing them not to voluntarily constrict their leg muscles during tilt. Glass-fiber sensor probes for measurements of SkBF were placed at the midpoints on the flexor side of the left forearm and the lateral of the left thigh and calf. In the heating condition, mean $T_{\mathrm{sk}}$ was slightly decreased to inhibit the increase of $T_{\text {core }}$ during the HUT.

Protocol 2: This protocol was performed to examine the effect of increased $T_{\text {core }}$ on the cutaneous vascular response to an increase of venous pressure in the calf. Eight female and two male volunteers participated in this protocol. Each subject wore a tube-lined water-perfused suit that covered the entire body except for the head, left arm, and right leg. Mean $T_{\text {sk }}$ was maintained at $\sim 33^{\circ} \mathrm{C}$ in normothermia. Sensor probes for SkBF measurements were placed on the left forearm and the right calf. A thigh cuff $15 \mathrm{~cm}$ wide was positioned just above the knee. A mercury-in-Silastic strain gauge was positioned at the point of maximum circumference of the right calf. In each thermal condition, we abruptly occluded the thigh at 20,30, 50, 70 , and $80 \mathrm{mmHg}$ in this order in five subjects and in the opposite order in the remaining subjects. This enabled us to study the vascular response and swelling pattern of the calf over a wide range of transmural venous pressures, such as those that occur during changes in posture or tilt [17]. That is, we used this method to examine the isolated effect of local reflex in vasoconstriction in the lower extremities during HUT. Between each occlusion pressure, the thigh cuff pressure was abruptly released to zero by opening the inflation bulb. The criterion for deflating the cuff was either (i) when the increase of calf circumference reached a steady-state level or (ii) when its increase was less than $0.5 \mathrm{~mm}$ for $30 \mathrm{~s}[17,18]$. The first was mainly used for the cuff pressures of 20,30 , and $50 \mathrm{mmHg}$, at which the calf circumference reached a steady state within 1-4 min. The latter criterion was mainly used for the higher cuff pressures, 70 and $80 \mathrm{mmHg}$, and the occlusion time ranged from 5 to $10 \mathrm{~min}$. Between reinflations of the cuff, we waited 2-3 min, or the time for the circumference to return to a steady-state baseline. After the cuff occlusion test was finished in normothermia, mean $T_{\text {sk }}$ was increased to $\sim 38^{\circ} \mathrm{C}$, and the same test was performed during whole-body heating. Although the water-perfused suit did not cover the head, left arm, and right leg in a subject, for convenience we used the term "whole-body heating" in this study.

Protocol 3: This protocol was performed to examine the effect of local warming on the cutaneous vascular response to an increase of venous pressure in the calf. Seven female and three male volunteers participated in this protocol. Each subject wore shorts and a T-shirt, and the right calf was inserted into a sealed box. During normothermic control, the air temperature in the box was maintained at 
$28-29^{\circ} \mathrm{C}$. After an evaluation of SkBF responses during the same cuff occlusion test as in protocol 2, the right calf was heated by increasing the air temperature in the box in the range of $42-48^{\circ} \mathrm{C}$. The $T_{\mathrm{sk}}$ in the right calf was maintained at $38-39^{\circ} \mathrm{C}$ during local heating. After the SkBF in the calf reached a steady state during local heating, the changes of SkBF during thigh occlusion were evaluated.

Measurements. In all protocols, SkBF was monitored continuously with laser-Doppler flow (LDF) meters (ALF21, Advance, Tokyo). The depth of LDF measurement was $\sim 1 \mathrm{~mm}$ from the skin surface, and the LDF is not influenced by blood flow to underlying skeletal muscle [19]. Blood pressure was recorded continuously from the left middle finger, using the Penaz principle (Finapres blood pressure monitor, Ohmeda, Madison, WI, USA) and a blood pressure unit (2238, NEC, Tokyo). This method permits accurate noninvasive measurement of arterial pressure during HUT [20]. The mean arterial pressure (MAP) expressed as $\mathrm{mmHg}$ was obtained from the electrical integration of the continuous pressure signal. HR was determined from the electrocardiogram $\left(\mathrm{CM}_{5}\right.$ lead). As indices of $T_{\text {core }}$, the sublingual or esophageal temperature was measured with a polyethylene-sealed thermocouple. Esophageal temperature was used in protocol 2. $T_{\text {sk }}$ was measured with copper-constantan thermocouples placed on the chest, upper back, lower back, abdomen, forearm, thigh, and calf, and mean $T_{\mathrm{sk}}$ was calculated [21].

In protocol 1, the left atrial diameter (LAD) (in $\mathrm{mm}$ ), as an index of cardiopulmonary baroreceptor loading [2224], was measured by two-dimensional and M-mode echocardiography (Logiq 400 pro, GE Yokogawa Medical Systems, Tokyo) with a $3.5 \mathrm{MHz}$ transducer. The images were obtained from the fourth left intercostal space in the parasternal short axis view at the level of the aortic valve at the end of the ventricular systole. Forearm blood flow (FBF) was determined by a venous occlusion plethysmography [25], with a Whitney mercury-in-Silastic strain gauge plethysmograph (Vasculab, model SPG16 Medsonics, Mountain View, CA, USA), every $20 \mathrm{~s}$ for the last 2 min at each angle of HUT. A strain gauge was placed on the subject's right midforearm, and two cuffs were secured; one on the upper arm and the other on the wrist. The venous occlusion cuff was placed just proximal to the elbow at a pressure of $50 \mathrm{mmHg}$, and the circulation in the hand was occluded by an inflation of the wrist cuff to a pressure of $250 \mathrm{mmHg}$. The forearms were always positioned at heart level during the HUT test.

In protocols 2 and 3, the changes in right calf circumference from the control value caused by cuff occlusion in the right thigh were determined with a gauge made of highly elastic tubing filled with mercury. The measured variables, except for LAD and FBF, were recorded by a data logger (DE1200 universal, NEC Sanei, Tokyo) every $5 \mathrm{~s}$.
Data analysis and statistics. The measured variables were averaged for the last $2 \mathrm{~min}$ at each angle of HUT. Forearm vascular conductance (FVC) was calculated as FBF/MAP and expressed as $\mathrm{ml} \cdot 100 \mathrm{ml}$ tissue ${ }^{-1} \cdot \mathrm{min}^{-}$ ${ }^{1}$. $\mathrm{mmHg}^{-1}$. The change in SkBF was expressed as a percent change from the supine control value during normothermia. Furthermore, to examine any effects of the increased SkBF under heating condition, we also expressed the change in SkBF as a percent change from the supine control value before HUT or cuff inflation during heating. Data were expressed as the mean \pm SE. The effects of tilt and thermal stress were evaluated with a two-way repeated measures ANOVA and the Bonferroni test. The effects of thigh cuff occlusion and thermal stress were also evaluated, using the two-way repeated measures ANOVA and Bonferroni test. Regression analysis was performed with the least squares methods, and comparisons of the slopes of linear relationships between vascular conductance and LAD were made with Student's paired $t$-tests. $P<0.05$ was considered significant.

\section{RESULTS}

\section{Protocol 1}

Changes in $T_{\text {core }}$ and cardiovascular variables during a graded HUT under normothermic and whole-body heating conditions are shown in Table 1. Whole-body heating increased $(P<0.001) T_{\text {core }}$. Moreover, whole-body heating increased $(P<0.001) T_{\mathrm{sk}}$ at the sites where SkBF was measured, from $32.6 \pm 0.2^{\circ} \mathrm{C}$ to $35.1 \pm 0.3^{\circ} \mathrm{C}$ in the forearm, from $33.3 \pm 0.2^{\circ} \mathrm{C}$ to $38.2 \pm 0.3^{\circ} \mathrm{C}$ in the thigh, and from $33.0 \pm 0.2^{\circ} \mathrm{C}$ to $35.7 \pm 0.4^{\circ} \mathrm{C}$ in the calf, respectively. In both thermal conditions, $T_{\text {core }}$ was not changed during HUT. LAD decreased with an increasing angle during tilt $(P<0.001)$ in both thermal conditions. Whole-body heating decreased $(P<0.001)$ LAD at each angle of tilt and increased $(P<0.001) \mathrm{FBF}$ and FVC. In each thermal condition, $\mathrm{FBF}$ and $\mathrm{FVC}$ decreased $(P<0.001)$ as the angle during the tilt test increased. Significant decreases in FVC with tilting were observed at an angle of $15^{\circ}$ or more in both thermal conditions. HUT increased $(P<0.01)$ HR and blood pressures as the angle increased in both thermal conditions. Whole-body heating increased $(P<0.001)$ HR, but did not change $(P>0.27)$ blood pressures.

Changes in SkBF during the HUT test are depicted in Fig. 1. Significant decreases in SkBF in the forearm with tilting were observed at an angle of $60^{\circ}$ during normothermia and at an angle of $45^{\circ}$ or more during heating (Fig. 1, $\mathrm{A}$ and $\mathrm{B})$. There were significant correlations in the changes of FVC to the decrease of LAD during HUT. Whole-body heating increased the slope $(0.24 \pm 0.06$ $\mathrm{ml} \cdot 100 \mathrm{ml}$ tissue ${ }^{-1} \cdot \mathrm{min}^{-1} \cdot 100 \mathrm{mmHg}-1 . \mathrm{mm}^{-1}$ in normothermia, and $0.65 \pm 0.10 \mathrm{ml} \cdot 100 \mathrm{ml}$ tissue ${ }^{-1} \cdot \mathrm{min}^{-1} \cdot 100$ 
Table 1. Changes in core temperature and cardiovascular variables during a graded head-up tilt (HUT) in both thermal conditions.

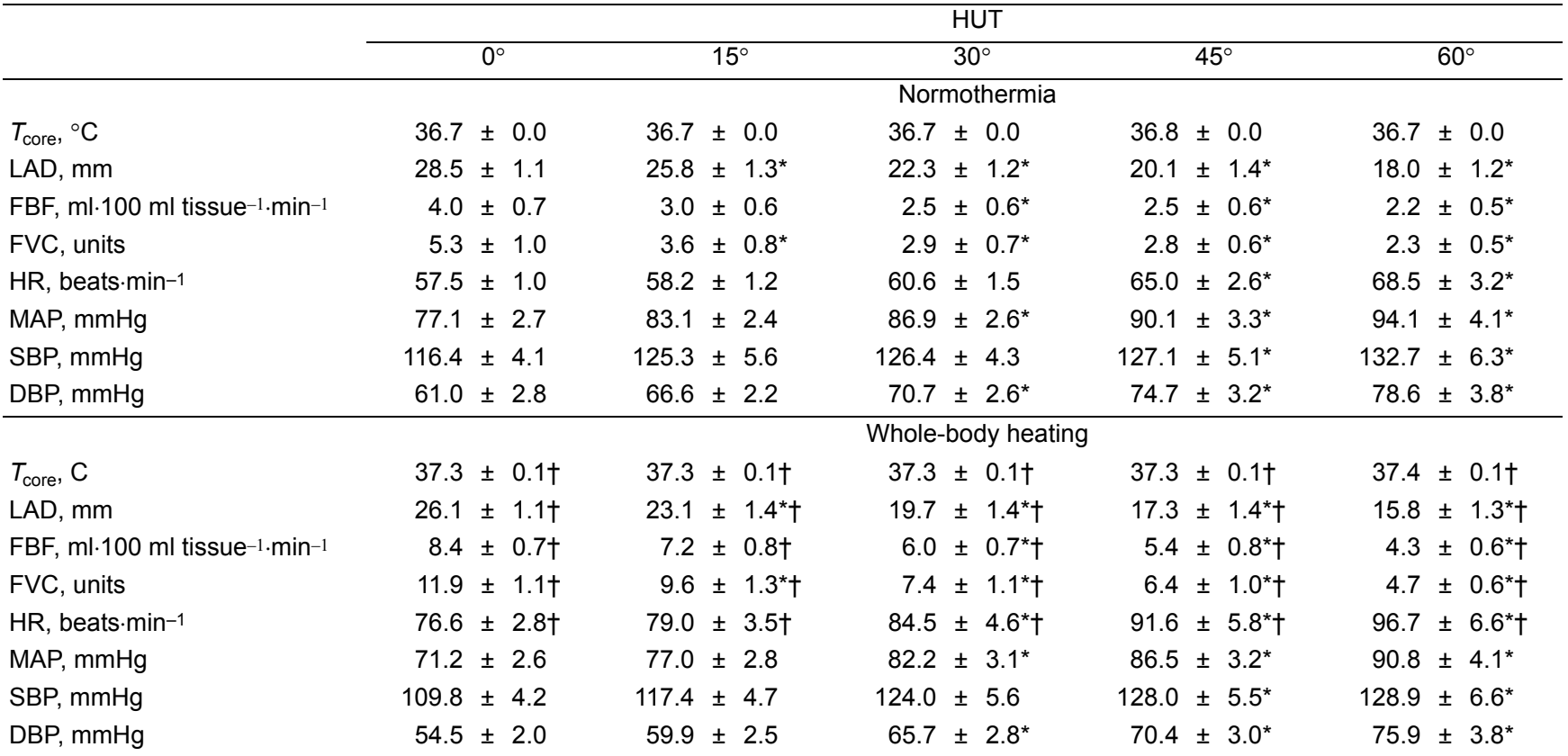

Values are means \pm SE. $T_{\text {core }}$, body core temperature; $L A D$, left atrial diameter; FBF, forearm blood flow; FVC, forearm vascular conductance; HR, heart rate; MAP, mean arterial pressure; SBP, systolic blood pressure; DBP, Diastolic blood pressure. The FVC is presented as ml. $100 \mathrm{ml}^{\mathrm{t}}$ tissue ${ }^{-1} \cdot \mathrm{min}^{-1} \cdot \mathrm{mmHg}^{-1} \times 100$. ${ }^{*} P<0.05$ vs. Supine control $\left(0^{\circ}\right) . \dagger P<0.05$ vs. Normothermia.

$\mathrm{mmHg}^{-1} \cdot \mathrm{mm}^{-1}$ in heating condition; $\left.P<0.001\right)$ in the relationship between FVC and LAD.

In normothermia, $\mathrm{SkBF}$ in the thigh and the calf decreased $(P<0.05)$ with tilting at $15^{\circ}$, then decreased gradually as the angle increased (Fig. 1, C-F), but during heating, SkBF at these sites decreased $(P<0.05)$ with tilting at an angle of $60^{\circ}$. That is, heat stress diminished the reduction of SkBF during HUT at the lower angles.

\section{Protocol 2}

Whole-body heating increased $(P<0.001) T_{\text {core }}$ from $36.9 \pm 0.1^{\circ} \mathrm{C}$ to $37.4 \pm 0.1^{\circ} \mathrm{C}$, and the elevated body temperature increased $(P<0.001) \mathrm{SkBF}$ in the calf by $295 \pm$ $98 \%$ and $\mathrm{SkBF}$ in the forearm by $401 \pm 74 \%$ from the baseline levels in normothermia, respectively, and the increase in calf SkBF did not differ from that in forearm SkBF $(P=0.72)$. During this heating maneuver, because the sites where $\mathrm{SkBF}$ were measured were not warmed, the $T_{\text {sk }}$ in the calf was maintained at $31-32^{\circ} \mathrm{C}$ throughout the experiment. Cuff inflation at the thigh did not change the systemic blood pressure and HR during normothemia and whole-body heating. These results suggest that the cuff inflation maneuver did not affect systemic vascular control under these conditions. In both thermal conditions, calf SkBF decreased $(P<0.001)$ with increasing cuff pressure at the thigh, but forearm SkBF was unchanged by inflation of the cuff. As shown in Fig. 2, the relationship between calf SkBF and cuff pressure showed an inverse S-shaped curve in both thermal conditions. Sig- nificant decreases of SkBF were observed at pressures of $20 \mathrm{mmHg}$ and above during normothermia and of 30 $\mathrm{mmHg}$ and above during whole-body heating, respectively. That is, whole-body heating shifted the response curve to the right (Fig. 2B).

\section{Protocol 3}

Local heating increased $(P<0.0001) T_{\mathrm{sk}}$ in the heated calf from $33.4 \pm 0.1^{\circ} \mathrm{C}$ to $38.5 \pm 0.2 \mathrm{C}$ without a significant change in $T_{\text {core }}$. Local heating of the calf increased $(P<$ $0.0001) \mathrm{SkBF}$ in the calf by $247 \pm 28 \%$ from the baseline level in normothermic control, but it did not change SkBF in the forearm. Calf SkBF decreased $(P<0.0001)$ with increasing cuff pressure at the thigh (Fig. 3), but forearm SkBF was not significantly changed by inflation of the cuff. Similar to the results in protocol 2, significant decreases of SkBF were observed at pressures of $20 \mathrm{mmHg}$ and above in the normothermic control and of $30 \mathrm{mmHg}$ and above during local heating, respectively. That is, local heating shifted the relationship between calf $\mathrm{SkBF}$ and cuff pressure to the right (Fig. 3B).

\section{DISCUSSION}

The major findings of the present study were as follows. First, in normothermia, HUT at the lower angles $\left(15^{\circ}\right.$ and $30^{\circ}$ ) did not change $\mathrm{SkBF}$ in the forearm, but it significantly decreased SkBF in the thigh and calf. Second, during whole-body heat stress, at an angle of $30^{\circ}$ or more 

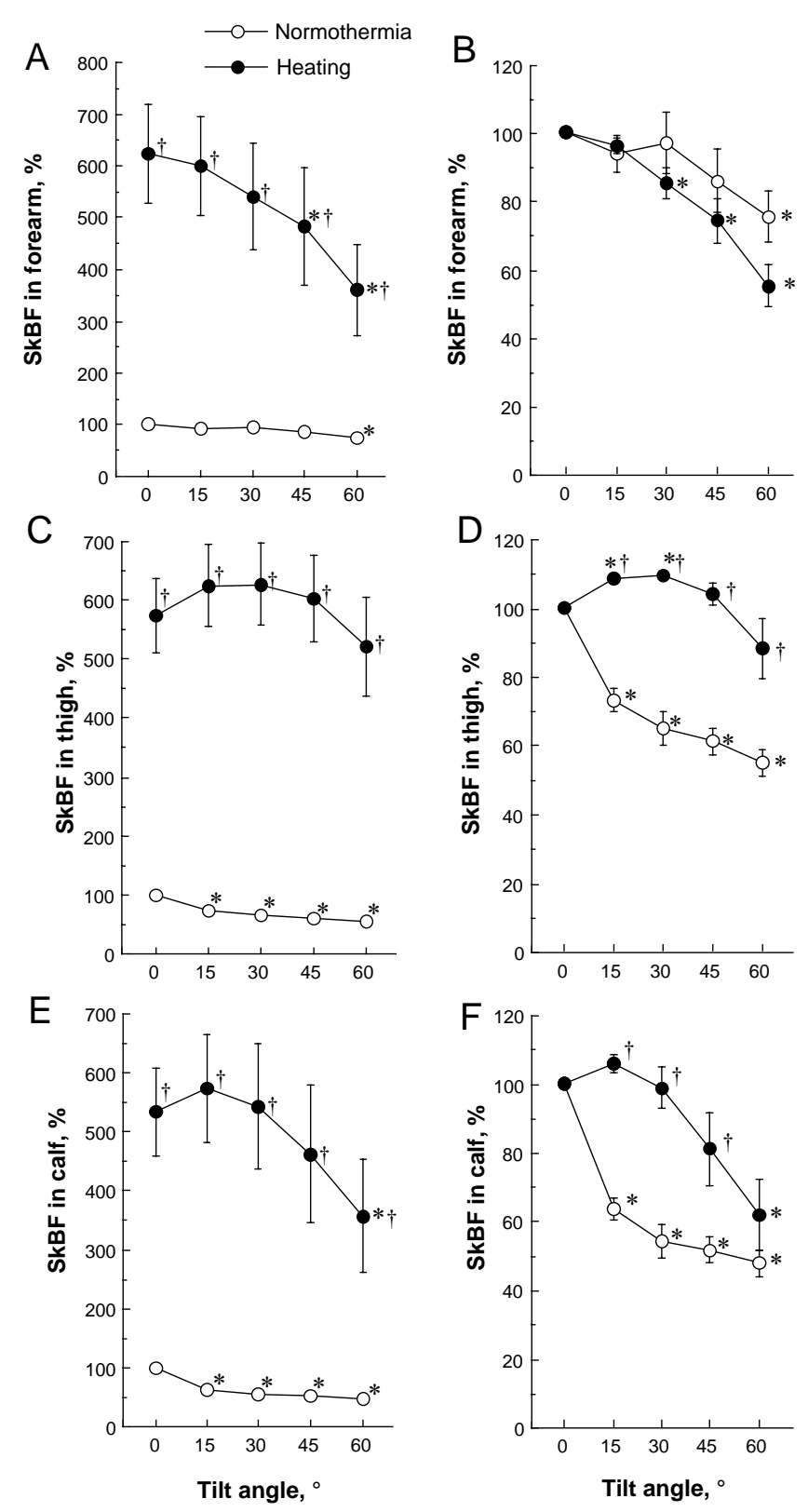

HUT significantly decreased SkBF in the forearm, but at the lower angles it did not change SkBF in the thigh or calf. Third, when whole-body and local heat stress similarly increased calf SkBF, the different heating maneuvers similarly increased venous pressure to induce a reduction of blood flow in the calf skin during cuff inflation at the thigh.

Under less orthostatic stress during normothermia, SkBF in the forearm positioned at heart level was unchanged, but SkBF in the leg was decreased significantly (Fig. 1). A reason for the regional difference in responses may be that vasoconstriction via baroreflex is greater in the legs than in the arms. In agreement with this concept, Vissing et al. [26] have reported that an increase in vascular resistance in the calf as a function of the decrease in central venous pressure produced by graded LBNP is larger than that in the forearm. On the contrary, Essandoh et
Fig. 1. Changes in skin blood flow (SkBF) in the forearm (Panels A, B), thigh (Panels C, D), and calf (Panels E, F) during a graded head-up tilt (HUT) test in protocol 1. Changes in SkBF are presented as percent changes from the normothermic control values (Panels $\mathbf{A}, \mathbf{C}, \mathbf{E}$ ) at $0^{\circ}$. Changes in SkBF during whole-body heating are also presented as percent changes from the pretilt values during heating (Panels B, D, F). ${ }^{*} P<$ 0.05 compared with the baseline control in each thermal condition, and $\dagger P<0.05$ compared to normothermia. Open and closed circles demonstrate data during normothermic control and whole-body heating, respectively.

al. [27] have reported that baroreflex-induced vasoconstriction was greater in the forearm than in the calf during mild LBNP at a level of $-20 \mathrm{mmHg}$ and lower. Thus the consensus of this concept is not still recognized. Furthermore, in the previous reports [26, 27], since blood flow was measured with plethysmography, it represents the composite of flow in both muscle and skin. To our knowledge, no report shows a marked baroreflex-induced cutaneous vasoconstriction in the lower extremities compared with the upper extremities.

As another reason for the regional difference in responses during normothermia, it is speculated that a local reflex function besides the integrated baroreflex function contributed to the vasoconstrictor response in the leg positioned below heart level during HUT. This response to an increase in venous pressure has been demonstrated in human cutaneous tissue $[8,28,29]$. It has been shown to be due to a local sympathetic reflex mechanism, the venoarteriolar reflex [3, 30, 31]. Crandall et al. [8] have reported that the venoarteriolar response in the skin did not occur through $\alpha$-adrenergic mechanisms and suggested that it is due to myogenic mechanisms associated with changes in vascular pressure, or it is a nonadrenergic, but neurally mediated, response. The mechanism for the venoarteriolar reflex is still unclear, but it is important that it is fully understood [32].

An interesting finding in this study is that whole-body heating diminished the reduction of SkBF in the leg during HUT at the lower angles $\left(15^{\circ}\right.$ and $\left.30^{\circ}\right)$. At least two mechanisms are postulated for the diminished vasoconstrictor response. First, it is possible that the vasoconstriction induced by baroreflex may be decreased by heat stress. However, a significant decrease in forearm SkBF from the pretilt level was obtained during HUT at the lower angles during whole-body heating compared to normothermia (Fig. 1, A and B). Moreover, the decreases in FVC with decreasing LAD during graded HUT were greater during whole-body heating than during normothermia. These results suggest that whole-body heating does not decrease baroreflex-induced vasoconstriction in the fore$\mathrm{arm}$. Therefore the impairment of baroreflex-induced vasoconstriction during heat stress may show a regional difference. 


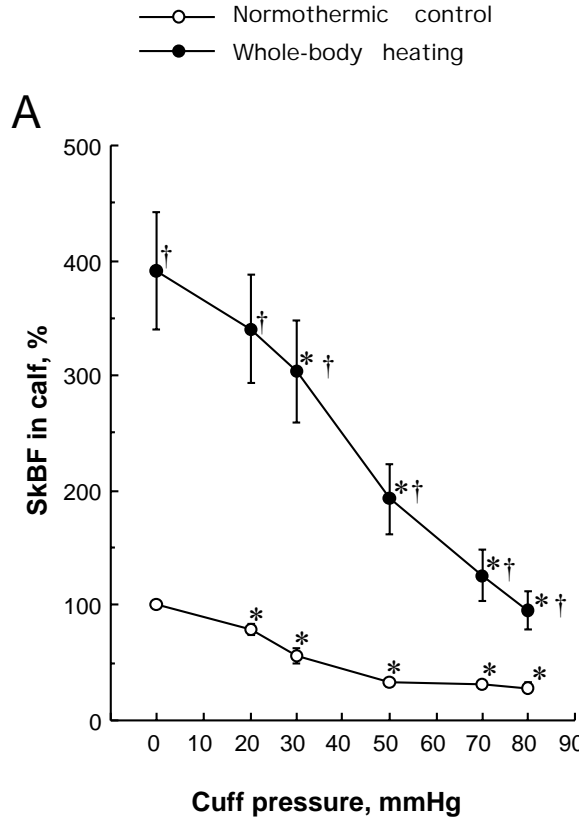

\section{B}

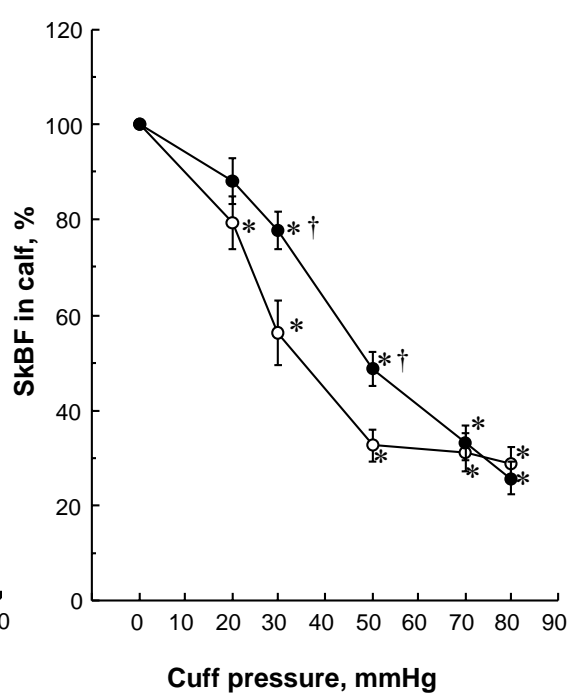

-

$\longrightarrow$ Local heating

A

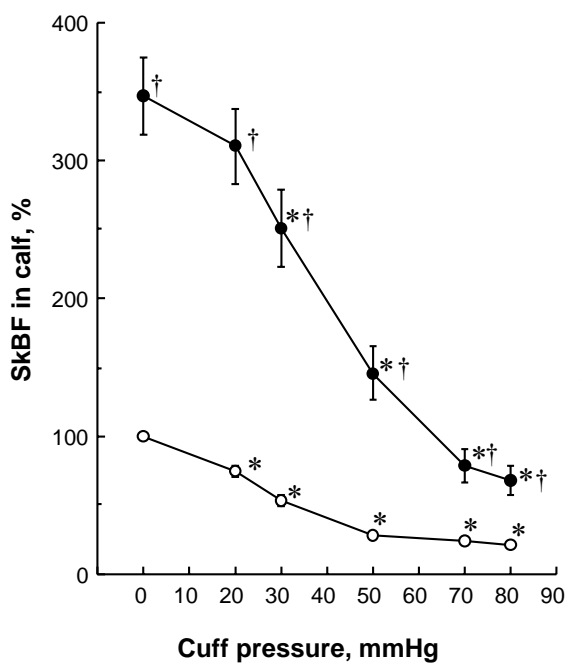

B

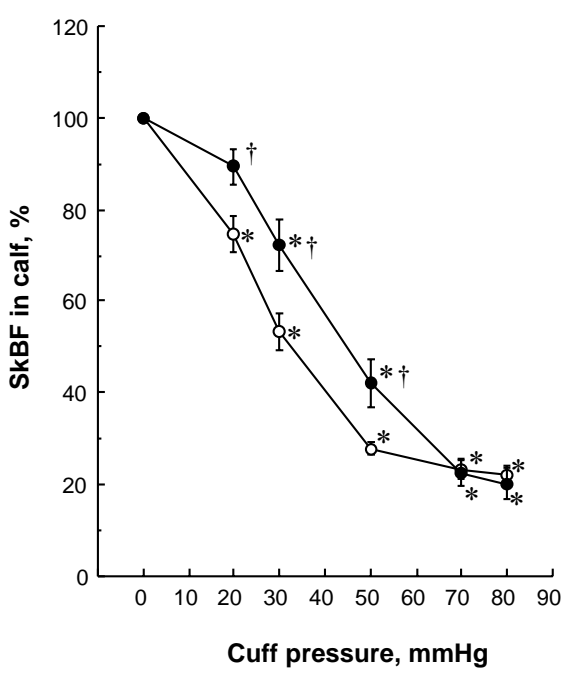

Fig. 2. Effect of whole-body heating on the changes in SkBF in the calf in response to changes in thigh cuff pressure in protocol 2. In protocol 2, skin temperatures at the sites where SkBF was measured were maintained at $31-32^{\circ} \mathrm{C}$. Changes in calf SkBF are presented as percent changes from the baseline control values under the normothermic condition (Panel A). Changes in SkBF during whole-body heating are also presented as percent changes from the baseline values during heating (Panel B). ${ }^{*} P<$ 0.05 compared with the baseline control in each thermal condition, and $\dagger P<0.05$ compared to normothermia. Open and closed circles demonstrate data during normothermic control and whole-body heating, respectively.

Fig. 3. Effect of local heating on the changes in calf SkBF in response to changes in thigh cuff pressure in protocol 3. Changes in calf SkBF are presented as percent changes from the baseline control values under the normothermic condition (Panel A). Changes in SkBF during local heating are also presented as percent changes from the baseline values during heating (Panel B). ${ }^{*} P<0.05$ compared with the baseline control in each thermal condition, and $\dagger P<0.05$ compared to normothermia. Open and closed circles demonstrate data during normothermic control and local heating, respectively an increased $T_{\text {core }}$ and whole-body $T_{\text {sk }}$ are not directly needed for heat-stress-induced modulation of the local reflex response. Thus it is thought that increased temperature itself is not essential, but that it is an inducible stimulation for heat-stress-induced modification of venoarteriolar response in the skin. It has been reported that the venoarteriolar response is abolished when blood flow is increased via local administration of histamine or sodium nitroprusside $[29,33]$. Therefore the heat-stressinduced modification of venoarteriolar response may depend on the increased blood flow in skin. It has been also reported that direct temperature effect on the venoarteriolar response is related to the attenuated response during local heating to $42^{\circ} \mathrm{C}$ [12]. The mechanism behind the heat-stress-mediated modulation of the local vascular re- control.

There are some caveats in this study. Since the laserDoppler flowmetry used monitors blood flow in the tissue near the skin surface (depth $=\sim 1 \mathrm{~mm}$ ), the prediction of LDF in a greater depth of skin tissue is difficult. Also, since the flowmetry does not discriminate SkBF in the artery and vein, a decreased perfusion pressure in the calf during thigh cuff occlusion might be, in part, a cause of the reduction in the calf SkBF. Cutaneous vascular conductance $(\mathrm{CVC})(=\mathrm{SkBF} /$ perfusion pressure) rather than $\mathrm{SkBF}$ is a better expression for an estimation of skin vasomotor response. But we did not present $\mathrm{CVC}$ in each site in this study because it is difficult to correctly estimate perfusion pressure in microvasculature during HUT or inflation of the cuff. The arterial pressure response during 
HUT under normothermic condition did not differ from that under heating condition. Thigh cuff inflation did not alter arterial pressure, and we analyzed steady-state data for SkBF responses to increasing cuff pressure under each thermal condition. It is thus thought that the different responses of SkBF during normothermia and heating largely indicated the difference in skin vasomotor responses. Therefore these technical limitations do not alter the interpretation that skin vasoconstrictor response is modified locally by the increased blood flow during heat stress.

HUT significantly increased blood pressure in protocol 1. This increase is not due to the pumping action in the leg muscle because subjects did not voluntarily constrict their leg muscle during HUT. It may have been due to visual or vestibular stimulation with tilting $[10,34,35]$. It is speculated that the visual or vestibular stimulation in addition to baroreceptor unloading might increase peripheral vascular resistance and thereby increase blood pressure during HUT. However, arterial blood pressure is not always increased by HUT [10, 11, 13-16, 37]. It is supposed that the changes in blood pressure during orthostatic stress depend on the duration, magnitude, and type (i.e., tilt, LBNP, or $\mathrm{Gz}$ centrifugation) of the gravitational stress and thermal condition.

There are documented studies of sex differences in cutaneous vascular response to heat stress [36, 37]. In protocols 2 and 3 , however, there was no clear difference in the $\mathrm{SkBF}$ responses between male and female subjects. It is thus speculated that heat stress similarly modulated the local vascular responses in both male and female subjects. Nevertheless, we should admit the potential limitation in this study. Further study is warranted for sex differences in local vascular responses in the skin.

In conclusion, the results from the present study suggest that SkBF in the lower extremities are more decreased during low orthostatic stress than those in the upper extremities at heart level during normothermia, and whole-body and local heat stress diminishes the skin vasoconstrictor response in the lower extremities with a locally altered vascular responsiveness.

The technical help of Ms. Y. Aoyagi, Ms. M. Hashimoto, and Ms. A. Hayashibara is greatly appreciated. Appreciation is also expressed to the participants in this study. This work was supported by a research grant from the University of Occupational and Environmental Health.

\section{REFERENCES}

1. Johnson JM, Proppe DW. Cardiovascular adjustments to heat stress. In: Fregly MJ, Blatteis CM, editors. Handbook of physiology. Section 4: Environmental physiology. Vol 1, New York (NY): Oxford University Press; 1996. p. 215-43.

2. Rowell LB. Cardiovascular adjustments to thermal stress. In: Shepherd JT, Abboud FM, editors. Handbook of physiology. Section 2: The cardiovascular system. Vol 3 pt 2. Bethesda (MD): American Physiological Society; 1983. p. 967-1023.

3. Rowell LB. Human circulation. Regulation during physical stress. New York: Oxford University Press; 1986.

4. Peters JK, Nishiyasu T, Mack GW. Reflex control of the cutaneous circulation during passive body core heating in humans. J Appl Physiol. 2000;88:1756-64.
5. Crandall CG, Johnson JM, Kosiba WA, Kellogg DL Jr. Baroreceptor control of the cutaneous active vasodilator system. J Appl Physiol. 1996;81:2192-8.

6. Johnson JM, Niederberger M, Rowell LB, Eisman MM, Brengelmann GL. Competition between cutaneous vasodilation and vasoconstrictor reflexes in man. J Appl Physiol. 1973;35:798-803.

7. Kellogg DL Jr, Johnson JM, Kosiba WA. Baroreflex control of cutaneous active vasodilator system in humans. Circ Res. 1990;66:1420-6.

8. Crandall CG, Shibasaki M, Yen TC. Evidence that the human cutaneous venoarteriolar response is not mediated by adrenergic mechanisms. J Physiol. 2002;538:599-605.

9. Vissing SF, Secher NH, Victor RG. Mechanisms of cutaneous vasoconstriction during upright posture. Acta Physiol Scand. 1997;159:131-8.

10. Watenpaugh DE, Breit GA, Buckley TM, Ballard RE, Murthy G, Hargens AR. Human cutaneous vascular responses to whole-body tilting, $\mathrm{Gz}$ centrifugation, and LBNP. J Appl Physiol. 2004;96:2153-60.

11. Yamazaki F, Monji K, Sogabe Y, Sone R. Cardiac and peripheral vascular responses to head-up tilt during whole body thermal stress. J UOEH. 2000;22:147-58

12. Davison JL, Short DS, Wilson TE. Effect of local heating and vasodilation on the cutaneous venoarteriolar response. Clin Auton Res. 2004;14:385-90.

13. Lind AR, Leithead CS, McNicol GW. Cardiovascular changes during syncope induced by tilting men in the heat. J Appl Physiol. 1968;25:268-76.

14. Shvartz E, Meyerstein N. Effect of heat and natural acclimatization to heat on tilt tolerance of men and women. J Appl Physiol. 1970;28:428-32

15. Shvartz E, Wyndham $\mathrm{CH}$, Strydom NB. Orthostatic responses in Caucasians and Bantu. Aviat Space Environ Med. 1975;46:1343-8.

16. Yamazaki F, Sone R. Thermal stress modulates arterial pressure variability and arterial baroreflex response of heart rate during head-up tilt. Eur J Appl Physiol. 2001;84:350-7.

17. Yamazaki F, Okuno C, Nagamatsu S, Sone R. Effects of whole-body and local thermal stress on hydrostatic volume changes in the human calf. Eur J Appl Physiol. 2002;88:61-6.

18. Melchior FM, Fortney SM. Orthostatic intolerance during a 13-day bed rest does not result from increased leg compliance. J Appl Physiol. 1993;74:286-92.

19. Saumet JL, Kellogg DL Jr, Taylor WF, Johnson JM. Cutaneous laser-Doppler flowmetry: influence of underlying muscle blood flow. J Appl Physiol. 1988;65:478-81.

20. Friedman DB, Jensen FB, Matzen S, Secher NH. Non-invasive blood pressure monitoring during head-up tilt using the Penaz principle. Acta Anaesthesiol Scand. 1990;34:519-22.

21. Taylor WF, Johnson JM, O'Leary D, Park MK. Effect of high local temperature on reflex cutaneous vasodilation. J Appl Physiol. 1984;57:191-6.

22. Greenleaf JE, Petersen TW, Gabrielsen A, Pump B, Bie P, Christensen NJ, Warberg J, Videbaek R, Simonson SR, Norsk P. Low LBNP tolerance in men is associated with attenuated activation of the renin-angiotensin system. Am J Physiol Regulatory Integrative Comp Physiol. 2000;279:R822-9.

23. Nabel EG, Colucci WS, Lilly LS, Cutler SS, Majzoub JA, St. John Sutton MG Dzau VJ, Creager MA. Relationship of cardiac chamber volume to baroreflex activity in normal humans. J Clin Endocrinol Metab. 1987;65:475-81.

24. Yamazaki F, Matsumura N, Nagata J, Ando A, Imura T. Spontaneous arterial baroreflex control of heart rate during head-down tilt in heat-stressed humans. Eur J Appl Physiol. 2001;85:208-13.

25. Whitney RJ: The measurement of volume changes in human limbs. J Physiol (London). 1953;121:1-27.

26. Vissing SF, Scherrer U, Victor RG. Relation between sympathetic outflow and vascular resistance in the calf during perturbations in central venous pressure. Evidence for cardiopulmonary afferent regulation of calf vascular resistance in humans. Circ Res. 1989;65:1710-7.

27. Essandoh LK, Houston DS, Vanhoutte PM, Shepherd JT. Differential effects of lower body negative pressure on forearm and calf blood flow. J Appl Physiol. 1986;61:994-8.

28. Henriksen O, Nielsen SL, Paaske WP, Sejrsen P. Autoregulation of blood flow in human cutaneous tissue. Acta Physiol Scand. 1973;89:538-43.

29. Henriksen $O$, Sejrsen P. Local reflex in microcirculation in human cutaneous tissue. Acta Physiol Scand. 1976;98:227-31.

30. Henriksen $\mathrm{O}$. Effect of chronic sympathetic denervation upon local regulation of blood flow in human subcutaneous tissue. Acta Physiol Scand. 1976;97:377-84.

31. Henriksen O, Alsner T. Effect of spinal sympathetic blockade upon local regulation of blood flow in subcutaneous tissue. Acta Physiol Scand. 1975;95:83-8.

32. Johnson JM. How do veins talk to arteries? J Physiol. 2002;538:341.

33. Zoltie N, Young C, Faris I, Tan E. The venoarteriolar reflex in free skin flaps. Clin 


\section{F. Yamazaki et al.}

Physiol. 1989:9:183-8.

34. Ray CA, Hume KM. Neck afferents and muscle sympathetic activity in humans: implications for the vestibulosympathetic reflex. J Appl Physiol. 1998;84:450-3.

35. Ray CA. Interaction of the vestibular system and baroreflexes on sympathetic nerve activity in humans. Am J Physiol Heart Circ Physiol. 2000;279:H2399-404

36. Inoue Y, Tanaka Y, Omori K, Kuwahara T, Ogura Y, Ueda H. Sex- and menstrual cycle-related differences in sweating and cutaneous blood flow in response to passive heat exposure. Eur J Appl Physiol. 2005;94:323-32.

37. Meendering JR, Torgrimson BN, Houghton BL, Halliwill JR, Minson CT. Menstrual cycle and sex affect hemodynamic responses to combined orthostatic and heat stress. Am J Physiol Heart Circ Physiol. 2005;289:H631-42. 